\title{
Determination of the complexity and diversity of the TCR $\beta$-chain CDR3 repertoire in bladder cancer using high-throughput sequencing
}

\author{
JINGSHENG MA ${ }^{1}$, GUOPING SUN ${ }^{2}$, PENG ZHU ${ }^{2}$, SONG LIU ${ }^{3}$, MINGLIN OU ${ }^{3}$, ZHIQIANG CHEN ${ }^{3}$, \\ CHANG ZOU ${ }^{3}$, FRANK LEING CHAN ${ }^{4}$, YONG DAI $^{3}$ and WEIGUO SUI ${ }^{1}$
}

\begin{abstract}
${ }^{1}$ Nephrology Department, Guangxi Key Laboratory of Metabolic Diseases Research, Guilin No. 181 Hospital (The Affiliated Guilim Hospital of Southern Medical University), Guilin, Guangxi 541002; ${ }^{2}$ Central Laboratory of Shenzhen Pingshan People's Hospital, Shenzhen, Guangdong 518118; ${ }^{3}$ Clinical Medical Research Center of The Second Clinical Medical College of Jinan University, Shenzhen People's Hospital, Shenzhen, Guangdong 518020; ${ }^{4}$ Cancer Biology and Experimental Therapeutics, School of Biomedical Sciences, The Chinese University of Hong Kong, SAR, P.R. China
\end{abstract}

Received March 8, 2018; Accepted December 6, 2018

DOI: $10.3892 / 01.2019 .10015$

\begin{abstract}
The present study aimed to investigate the complexity and diversity of the Tlymphocyte immune repertoire in patients with bladder cancer. To do so, the immune state of patients was assessed. The study also aimed to elucidate the aetiology and pathogenesis of bladder cancer to provide a novel theoretical basis for disease prevention, diagnosis, treatment and prognosis monitoring. Cancerous and paracancerous (control) tissue samples were collected from five patients diagnosed with muscle-invasive bladder cancer. Multiplex polymerase chain reaction and Illumina high-throughput sequencing were used to determine the characteristics and clonal diversity of the T-cell receptor (TCR) $\beta$-chain complementarity-determining region 3 (CDR3) gene in the cancerous and paracancerous tissues of patients with bladder cancer. The degree of clonal expansion in malignant samples was significantly higher than in adjacent samples. Furthermore, TCR $\beta$ variable (TRBV), TCR $\beta$ diversity (TRBD) and TCR $\beta$ joining (TRBJ) repertoires were significantly different in cancerous samples compared with adjacent samples. In addition, 13 identified V-J pairs were highly expressed in cancerous samples whereas they had low
\end{abstract}

Correspondence to: Dr Yong Dai, Clinical Medical Research Center of The Second Clinical Medical College of Jinan University, Shenzhen People's Hospital, 1017 Dongmen North Road, Luohu, Shenzhen, Guangdong 518020, P.R. China

E-mail: daiyong22@aliyun.com

Dr Weiguo Sui, Nephrology Department, Guangxi Key Laboratory of Metabolic Diseases Research, Guilin No. 181 Hospital (The Affiliated Guilim Hospital of Southern Medical University), 1 Xinqiaoyuan Road, Xiangshan, Guilin, Guangxi 541002, P.R. China

E-mail: suiwg@163.com

Key words: bladder cancer, high-throughput sequencing, immune repertoire, tumour-infiltrating lymphocytes, T-cell receptor expression in control samples. In conclusion, the degree of T-cell clonal expansion in bladder cancerous tissue was higher than in paracancerous tissue, whereas the immune diversity of the tissues of patients with bladder cancer was significantly lower. The DNA sequence and amino acid sequences, and V-J combination level may be used to comprehensively understand the diversity and characteristics of TCR CDR3 in bladder cancer and paracancerous tissues, and to evaluate the immune status of bladder cancer to develop therapeutic targets and biomarkers for prognosis monitoring.

\section{Introduction}

Increasing evidence has revealed that the adaptive immune response to cancer is a prospective prognostic marker and a diagnostic criterion for various types of cancer $(1,2)$. Previously, targeted immune-based therapies have revealed durable responses and may represent novel promising treatments for various types of cancer, including melanoma (3), breast (4), renal (5), non-small cell lung (6) and bladder cancer (7). These emerging immunotherapies, including the immunomodulators Bacillus Calmette-Guérin (BCG) polysaccharide nucleic acid and adoptive cell transfer, highlight the crucial need to better monitor and comprehend tumour-infiltrating lymphocytes (TILs).

$\mathrm{T}$ cells in cancer tissues are generated during $\mathrm{T}$-cell differentiation in the thymus (8) and serve a central role in the tumour immune response. $T$ cells recognize antigens displayed on the surface of cancer cells via the membrane protein T-cell receptor (TCR). Each T cell commonly possesses a single TCR. The majority of TCRs contain an $\alpha$-polypeptide chain and a $\beta$-polypeptide chain. Each chain has a variable region ( $\mathrm{V}$ region), a joining region ( $\mathrm{J}$ region) and a constant region (C region). The $\beta$ chain also possesses a diversity region (D region) (8). The $\mathrm{V}$ region of the TCR $\alpha$ - and $\beta$-chains contains three hypervariable regions, including complementarity determining regions (CDR) 1, 2 and 3, of which CDR3 is the most variable and therefore the primary source of 
antigen-specific recognition. The diversity of TCR is generated by a random rearrangement of the $\mathrm{V}, \mathrm{D}, \mathrm{J}$ and $\mathrm{C}$ regions, extended by non-germline-encoded nucleotides randomly inserted at the junctions of the final segments (9).

Since the diversity of distinct TCRs is $\sim 2.5 \times 10^{18}$ in a normal adult, the TCR repertoire is therefore difficult to analyse (10). High-throughput sequencing (HTS) is a novel method that can profile the TCR repertoire and analyse the adaptive immune response; therefore allowing the study of TCR repertoire diversity at a greater depth than previously $(11,12)$. Notably, this technology has been used to investigate and monitor the implication of TCR in tumour immunity through large-scale sequencing of CDR3 (13-15). The present study aimed to evaluate the complexity and diversity of cancer tissues compared with paracancerous tissues by amplifying the CDR3 of TCRs using multiplex polymerase chain reaction (PCR) and HTS. Decrypting the TCR $\beta$ chain repertoire in TILs may offer novel insights for the development of future biomarkers and monitoring in bladder cancer.

\section{Materials and methods}

Patients. Five patients with muscle-invasive bladder cancer were selected, and cancer and paracancerous specimens were collected at The Shenzhen People's Hospital (Shenzhen, China) between January and April 2016. The diagnosis of bladder cancer was confirmed by pathological diagnosis and clinical evidence. The patient cohort was 44-82 years old, with a mean age of $63.10 \pm 19.21$ years. These patients had not been treated with chemotherapy prior to the study, and written informed consent was obtained for study participation. This study passed the discussion and approval of the Ethics Committee of Guilin No. 181 Hospital (Guilin, China) and the Shenzhen People's Hospital (Shenzen, China).

Sample preparation. A total of 5-10 cancerous and paracancerous sections were obtained from each patient with bladder cancer, and total DNA was extracted using a standard method. Briefly, dewaxing was performed using xylene, followed by an overnight proteinase $\mathrm{K}$ digestion at $56^{\circ} \mathrm{C}$. Total DNA in tissue samples was extracted using the QIAamp DNA Mini kit (Qiagen GmbH, Hilden, Germany) according to the manufacturer's protocol. The DNA concentration of each sample was determined using a Qubit fluorometer (Invitrogen; Thermo Fisher Scientific, Inc., Waltham, MA, USA). DNA quality was evaluated via $0.8 \%$ agarose gel electrophoresis. The DNA samples extracted from the cancerous tissues of the five patients were mixed together in a 1:1:1:1:1 ratio according to the Qubit value, to obtain a unique cancer sample. Similarly, the DNA samples extracted from the paracancerous tissues of the five patient were mixed together in a 1:1:1:1:1 ratio according to the Qubit value, to obtain a unique control sample.

Multiplex polymerase chain reaction (PCR) amplification of the TCR $\beta C D R 3$ region. The human $\mathrm{TCR} \beta$ sequences were downloaded from the international ImMunoGeneTics information system ${ }^{\circledR}$ for immunoglobulins or antibodies (http://www.imgt.org/) (16). A relatively conserved region in frame region 3 , upstream of CDR3, was selected for the putative forward primer region. A cluster of primers (Table I) corresponding to the majority of the $\mathrm{V}$ gene sequence family was selected. Reverse primers, corresponding to the $\mathrm{J}$ gene family, were similarly designed (17). A total of 30 forward primers and 13 reverse primers were used with the QIAGEN multiplex PCR kit (Qiagen $\mathrm{GmbH}$ ) to amplify the rearranged TCR $\beta$ CDR3 region. The PCR reactions $(50 \mu \mathrm{l})$ consisted of $2 \mu \mathrm{l}$ TCR $\beta$ variable (TRBV) pool $(10 \mu \mathrm{M}), 2 \mu \mathrm{l}$ TCR $\beta$ joining (TRBJ) pool $(10 \mu \mathrm{M}), 25 \mu \mathrm{l} 2 \mathrm{X}$ QIAGEN Multiplex PCR Master, $5 \mu 15 \mathrm{X}$ Q-solution, $10 \mu \mathrm{l}$ template DNA and $6 \mu 1$ $\mathrm{H}_{2} \mathrm{O}$. The PCR conditions were $95^{\circ} \mathrm{C}$ for $15 \mathrm{~min}$ followed by 25 cycles of $94^{\circ} \mathrm{C}$ for $15 \mathrm{sec}$ and $60^{\circ} \mathrm{C}$ for $3 \mathrm{~min}$, with a final extension for $10 \mathrm{~min}$ at $72^{\circ} \mathrm{C}$. The PCR products were purified from the primer sequences by AMPure XP beads (Beckman Coulter, Inc., Brea, CA, USA). A second round of PCR was performed to add a sequencing index to each sample. The PCR reaction $(50 \mu \mathrm{l})$ consisted of $13.5 \mu \mathrm{l} \mathrm{H} \mathrm{H}_{2} \mathrm{O}, 0.5 \mu \mathrm{l} 2 \mathrm{X}$ Q5 DNA polymerase, $10 \mu \mathrm{l}$ 5X Q5 buffer, $1 \mu \mathrm{l}$ dNTP (10 mM), $1 \mu 1$ P1 $(10 \mu \mathrm{M}), 23 \mu \mathrm{l}$ DNA, and $1 \mu \mathrm{l}$ index $(10 \mu \mathrm{M})$. The PCR condition was set at $98^{\circ} \mathrm{C}$ for $1 \mathrm{~min}$, followed by 25 cycles of $98^{\circ} \mathrm{C}$ for $20 \mathrm{sec}, 65^{\circ} \mathrm{C}$ for $30 \mathrm{sec}$ and $72^{\circ} \mathrm{C}$ for $30 \mathrm{sec}$, with a final extension for $5 \mathrm{~min}$ at $72^{\circ} \mathrm{C}$. The library was separated by agarose gel electrophoresis, and the target region was isolated and cleaned using QIAquick Gel Extraction kits according to the manufacturer's protocol (Qiagen $\mathrm{GmbH}$ ).

High-throughput sequencing and data analysis. The library was quantified using an ABI StepOnePlus Real-Time PCR system (Applied Biosystems; Thermo Fisher Scientific, Inc.) and an Agilent 2100 Bioanalyzer instrument (Agilent DNA 1000 Reagents; Agilent Technologies GmbH, Waldbronn, Germany), and sequenced by Illumina MiSeq (Illumina, Inc., San Diego, CA, USA). Briefly, the low-quality reads and the adaptor reads were filtered from the raw data, and the clean data were used in further alignment. Subsequently, the clean data were aligned to the human TRBV database and were analysed using the online IMGT (ImMunoGeneTics)/HighV-QUSET and miTCR (MiLaboratory LLC, Solana Beach, CA, USA) tools. The data included the V, D and J assignments, the CDR3 length distribution, clustering, highly expanded clone and Shannon entropy index (18). Shannon entropy index is a measure of immune diversity in the field of immune library. This index ranges between 0 and 1, where 1 indicates the most diversity, and 0 indicates no diversity at all. The flow chart of biological information is presented in Fig. 1.

\section{Results}

T-cell receptor $\beta$ sequence statistics. A total of 1,789,531 raw reads and 857,022 raw reads were obtained for the cancer sample and the control sample, respectively. The miTCR was used to map the reads to the available databases. The mapping of the immune sequence reads, the total CDR3 sequences, and the unique CDR3 nucleotide sequences per sample are presented in Tables II and III.

Degree of expansion and diversity analysis of T-cell clones. In order to comprehensively and accurately understand the diversity of the TCR clones in the cancer and control samples, the degree of clonal amplification of the DNA sequences was calculated in each sample. The statistical method used to 
Table I. TRB V primers (5'-3').

\begin{tabular}{ll}
\hline Primer & \multicolumn{1}{c}{ Sequence } \\
\hline TRBV2 & ATTTCACTCTGAAGATCCGGTC \\
TRBV3-1 & CAC \\
& AAACAGTTCCAAATCGMTTCT \\
TRBV4-1/2/3 & CAC \\
TRBV5-1 & GCCAGTTCTCTAACTCTCGC \\
& TCT \\
TRBV5-4/5/6/8 & TCAGGTCGCCAGTTCCCTAAY \\
& TAT \\
TRBV6-4.1 & CACGTTGGCGTCTGCTGTAC \\
& CCT \\
TRBV6-8/5/1.2 & CAGGCTGGTGTCGGCTGCTC \\
TRBV6-9/7/1.1/6 & CCT \\
& CAGGCTGGAGTCAGCTGCTC
\end{tabular}

TRBV6-4.2

TRRBV6-2/3

TRBV7-2/4/6/7/8

TRBV7-3

TRBV7-9

TRBV9

TRBV10-1

TRBV10-2/3

TRBV11-1/2/3

TRBV12-3.2/5.2

TRBV12-3.1/4/5.1

TRBV13

TRBV14

TRBV15

TRBV16

TRBV18

TRBV19

TRBV20-1

TRBV24-1

TRBV25-1

TRBV27-1
AGTCGCTTGCTGTACCCTCT

CAG

GGGGTTGGAGTCGGCTGCTC

CCT

GGGATCCGTCTCCACTCTGAM

GAT

GGGATCCGTCTCTACTCTGAA

GAT

GGGATCTTTCTCCACCTTGGA

GAT

CCTGACTTGCACTCTGAACTAA

ACCT

CCTCACTCTGGAGTCTGCTGCC CCTCACTCTGGAGTCMGCT

ACC

GCAGAGAGGCTCAAAGGAGT

AGACT

GAAGGTGCAGCCTGCAGAAC

CCAG

GAAGATCCAGCCCTCAGAACC

CAG

TCGATTCTCAGCTCAACAGTTC

GGAGGGACGTATTCTACTCTGA

AGG

TTCTTGACATCCGCTCACCAGG

CTGTAGCCTTGAGATCCAGGC

TACGA

TAGATGAGTCAGGAATGC

CAAAG

TCCTTTCCTCTCACTGTGACAT

CGG

AACCATGCAAGCCTGACCTT

CTCCCTGTCCCTAGAGTCTGC

CAT

GCCCTCACATACCTCTCAGTA

CCTC

GATCCTGGAGTCGCCCAGC
Table I. Continued.

\begin{tabular}{ll}
\hline Primer & \multicolumn{1}{c}{ Sequence } \\
\hline TRBV28 & ATTCTGGAGTCCGCCAGC \\
TRBV29-1 & AACTCTGACTGTGAGCAACATGAG \\
TRBV30-F5 & CAGATCAGCTCTGAGGTGCCCCA \\
\hline
\end{tabular}

TRB, T-cell receptor $\beta$.

determine the degree of clonal amplification of the sequence in each sample represented the number of times the sequence was present in the test sample. In the present study, a highly expanded clone (HEC) was defined as a TCR clone with a frequency $>0.5 \%$ of the total reads in each sample. In the cancer sample, 32 DNA sequences were observed and defined as HECs, with a HEC ratio of 0.46 . Conversely, only 14 clones were HECs, with a HEC ratio of 0.19 , in the control sample. Subsequently, a plot of the frequency distribution of the TCR repertoire was developed in cancer and control samples. Few DNA sequences were present at substantially higher frequencies $(>1 \%)$, and the majority of the DNA sequences occurred at low frequencies in both cancer and control samples. The percentages of different abundance clones were determined. In the cancer sample, the percentage of clones with an abundance of $0.1-1 \%$ was $81.50 \%$ of the total T-cell sequences, whereas the percentage of clones with an abundance of $0.01-0.1 \%$ was $12.80 \%$. Conversely, in the control sample, the percentage of clones with an abundance of $0.1-1 \%$ was $32.60 \%$, whereas the percentage of clones with an abundance of 0.01-0.1\% was highest, at $43.86 \%$ (Fig. 2). The TCR repertoire in the cancer sample had a greater HEC value $(>0.5 \%)$, HEC ratio and a higher degree clone percentage $(>0.1 \%)$ than the control sample. To compare the TCR diversity of the cancer and control samples, the normalized Shannon entropy index was also used. The normalized Shannon entropy index of the cancer sample was lower than that of the control sample $(0.40$ vs. 0.59) (data not shown). The present study suggested that the entire TCR repertoire in the cancer sample had a substantially more skewed clonotype composition than the control sample.

Special and shared TCR sequences. To further assess how TCR sequences were shared between the cancer and control samples, deep sequencing was used to characterize the HEC sequences of the respective TCR repertoires. The analysis focused mainly on the DNA and amino acid (aa) sequences of CDR3, which was the most diverse region of the TCR molecule and was relevant to antigen epitope recognition. With regards to the DNA sequences, 12,516 special DNA sequences were obtained in the cancer sample, of which five were HECs. A total of 25,371 special DNA sequences were obtained in the control sample, of which 10 were HECs (Table IV). For the aa sequence, 9,424 special aa sequences were obtained in the cancer sample, of which, seven were HECs, whereas the control sample exhibited 21,020 special aa sequences, of which 10 were HECs (Table V). In addition, 680 shared DNA sequences and 701 shared aa sequences were determined in the cancer and control samples. In a further comparative analysis, all 


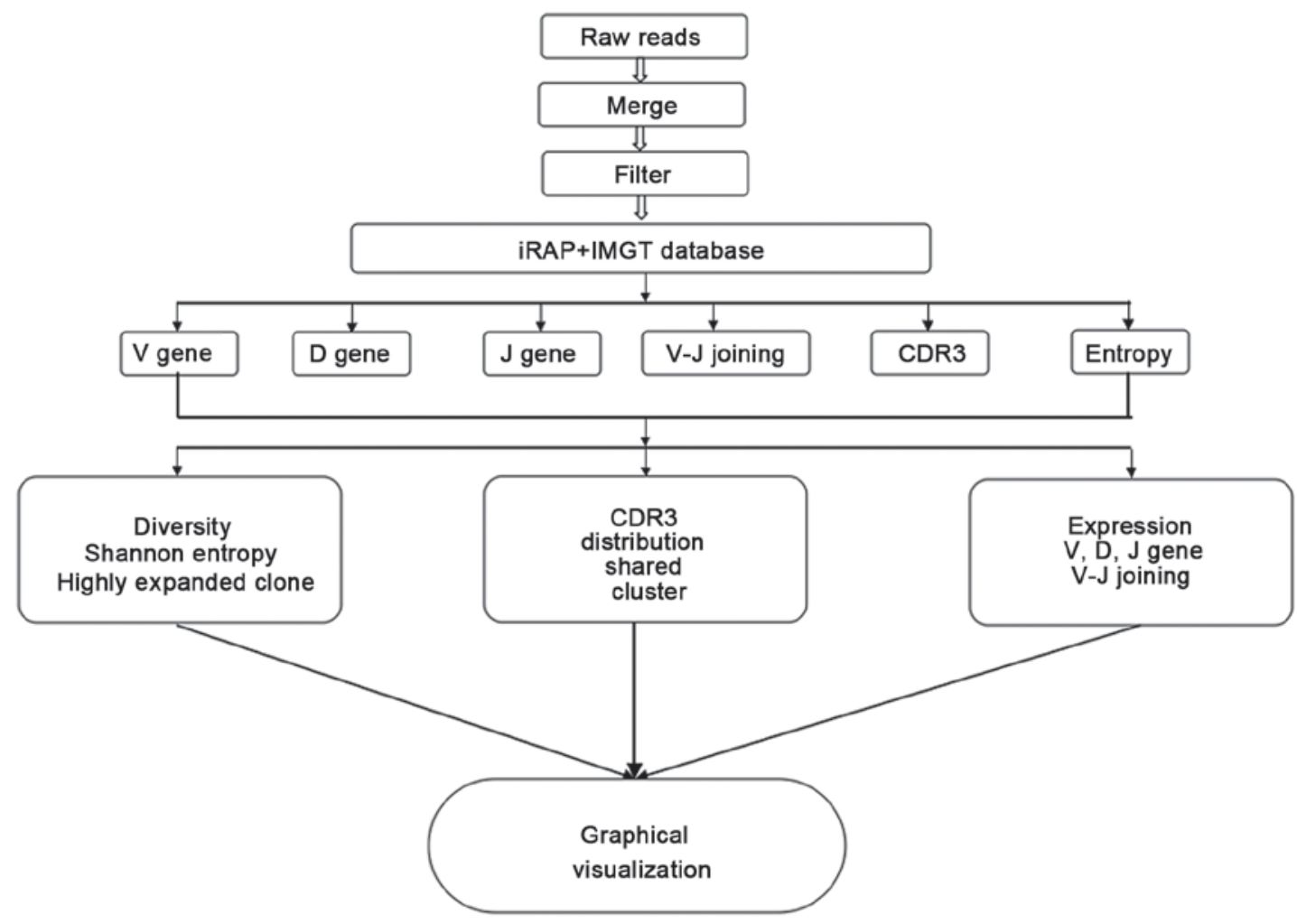

Figure 1. The flow chart of biological information analysis. CDR3, complementarity-determining region 3; IMGT, ImMunoGeneTics; iRAP, integrated RNS-seq analysis pipeline.

Table II. TRB J primers (5'-3').

\begin{tabular}{ll}
\hline Primer & \multicolumn{1}{c}{ Sequence } \\
\hline TRBJ1.1 & CTTACCTACAACTGTGAGTCTGGTG \\
TRBJ1.2 & CTTACCTACAACGGTTAACCTGGTC \\
TRBJ1.3 & CTTACCTACAACAGTGAGCCAACTT \\
TRBJ1.4 & AAGACAGAGAGCTGGGTTCCACT \\
TRBJ1.5 & CTTACCTAGGATGGAGAGTCGAGTC \\
TRBJ1.6 & CATACCTGTCACAGTGAGCCTG \\
TRBJ2.1 & CCTTCTTACCTAGCACGGTGA \\
TRBJ2.2 & CTTACCCAGTACGGTCAGCCT \\
TRBJ2.3 & CCGCTTACCGAGCACTGTCAG \\
TRBJ2.4 & AGCACTGAGAGCCGGGTCC \\
TRBJ2.5 & CGAGCACCAGGAGCCGCGT \\
TRBJ2.6 & CTCGCCCAGCACGGTCAGCCT \\
TRBJ2.7 & CTTACCTGTGACCGTGAGCCTG
\end{tabular}

TRB, T-cell receptor $\beta$.

27 HEC DNA sequences in the cancer sample demonstrated a lower expression $(<0.5 \%)$ in the control sample. Conversely, four HEC DNA sequences in the control sample had a lower expression $(<0.5 \%)$ in the cancer sample (Table VI). For the shared aa sequence, all $28 \mathrm{HECs}$ in the cancer sample exhibited a lower expression $(<0.5 \%)$ in the control sample. In contrast, four HECs in the control sample showed a lower expression $(<0.5 \%)$ in the cancer sample (Table VII). There were no shared
Table III. T-cell receptor $\beta$ sequence statistics.

\begin{tabular}{lrr}
\hline Data type & Cancer & Control \\
\hline Total raw reads & $1,789,531$ & 857,022 \\
Total clean sequences number & $1,081,491$ & 551,870 \\
Immune sequences number & $1,059,138$ & 543,038 \\
Unknown sequences number & 22,353 & 8,832 \\
Productive sequences number & 744,400 & 417,942 \\
Nonproductive sequences number & 314,738 & 125,096 \\
In-frame sequences number & 763,882 & 433,569 \\
Out-of-frame sequences number & 287,038 & 105,628 \\
Total CDR3 sequences number & 718,791 & 410,030 \\
Unique CDR3 nt sequences number & 13,196 & 26,051 \\
Unique CDR3 aa sequences number & 10,125 & 21,721 \\
\hline
\end{tabular}

aa, amino acid; CDR3, complementary-determining region 3; nt, nucleotide.

HECs in the DNA and aa sequences between the cancer and control samples. These results suggested that comparisons of the TCR HECs in cancer and control samples may accelerate the screening process for possible novel biomarkers.

Comparison of $T R B V, T C R \beta$ diversity (TRBD) and TRBJ repertoires. To determine whether cancer-specific differences exist in the TRBV, TRBD and TRBJ repertoires, the expression levels of the respective TRBV, TRBD and TRBJ repertoires were compared between the cancer and control 
Table IV. A summary of the highly expressed special DNA sequences $(>0.5 \%)$.

A, Cancer

Clonotype (5'-3')

Frequency $(\%)$

GCCACCAGTGAGTTCGACAGGGGTAAAGAGACCCAGTAC

0.54

GCCACCAGTGATCAGATTCATGTGGAGACCCAGTAC

0.71

GCCAGCAATACCGGGACAGGGGCATATGGCTACACC

0.72

GCCAGCAGTGATTTGGGGCCAGGCCTGTATGGCTACACC

0.77

GCCAGCTACTCCTATGGCTACACC

0.72

B, Control

Clonotype (5'-3')

Frequency $(\%)$

GCCACCAGCAAAGGGACGACTAGGGCCGGCGAGCAGTAC

GCCACCAGTTTTATTAAACAGCCCCAGCAT

1.49

GCCAGCAGCCCGGGACAACCACCCCTCCAC

0.59

GCCAGCAGCTTAGAGTCCGGACCCTACGAGCAGTAC

0.51

GCCAGCAGTGACAACGGGCCAAATCAGCCCCAGCAT

1.41

GCCAGCAGTGACCGGGGTGAGCAGTAC

1.03

GCCAGCAGTTACAGGGGGGGGGAGCTGTTT

1.35

GCCAGTAGCCCCACGGGGGCAGGGAACACTGAAGCTTTC

1.06

GCCTCCACCGACAACAGGGAGACCCAGTAC

1.41

GCCTGGAGTGTTACCGGGGCAGATGAAAAACTGTTT

0.62

Table V. A summary of the highly expressed special amino acid sequences $(>0.5 \%)$.

\begin{tabular}{lc}
\hline A, Cancer & \\
\hline Clonotype (5'-3') & Frequency (\%) \\
\hline ASNTGTGAYGYT & 0.73 \\
ASSDLGPGLYGYT & 0.78 \\
ASSMGRTDTQY & 0.53 \\
ASSVEGRTQY & 0.50 \\
ASYSYGYT & 0.72 \\
ATSDQIHVETQY & 0.73 \\
ATSEFDRGKETQY & 0.54 \\
\hline
\end{tabular}

B, Control

Clonotype (5'-3')

Frequency (\%)

ASSDNGPNQPQH

ASSDRGEQY

1.43

ASSLESGPYEQY

1.05

ASSPGQPPLH

0.51

ASSPTGAGNTEAF

0.60

1.08

ASSYRGGELF

1.37

ASTDNRETQY

1.42

ATSFIKQPQH

1.51

ATSKGTTRAGEQY

0.92

AWSVTGADEKLF

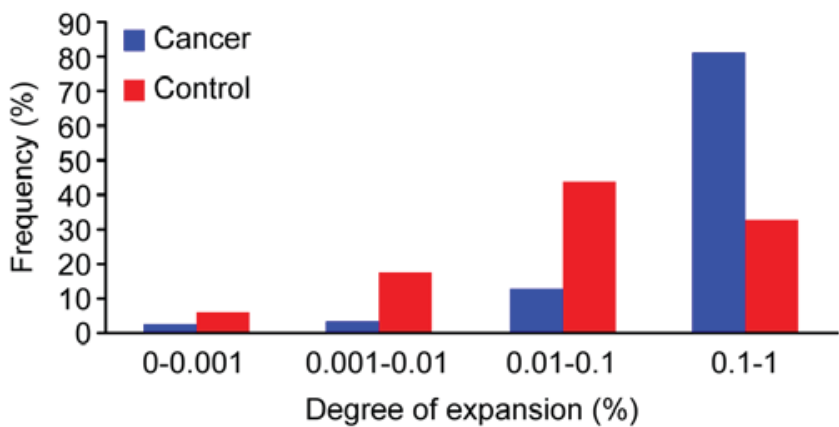

Figure 2. Degree of expansion and frequency distribution of the T-cell clones. The $\mathrm{x}$-axis depicts the degree of expansion; the $\mathrm{y}$-axis depicts the percentage of each clone frequency.

samples. Results demonstrated that four special TRBV segments (TRBV12-1, TRBV5-7, TRBV6-7 and TRBV6-8) were present in the control sample, whereas none were present in the cancer sample. For the 52 shared TRBV gene segments, the usage frequencies of TRBV2 (14.32 vs. 14.61\%) and TRBV25-1 (4.43 vs. $4.04 \%)$ were similar $( \pm 10 \%)$ between the cancer and control samples. For the other distinct TRBV gene segments, the usage frequencies of each TRBV gene exhibited extreme variation. A stacked bar chart was generated to display the frequencies of the 20 top TRBV genes, and differences were revealed between the cancer and control samples (Fig. 3). The usage frequencies of the TRBD gene segment were 38.42 and $61.58 \%$ for TRBD1 and TRBD2, respectively in the cancer sample. Conversely, the usage frequencies of the TRBD1 and TRBD2 gene segments were 53.76 and $46.24 \%$, respectively 
Table VI. A summary of the highly expressed shared DNA sequences $(>0.5 \%)$.

\begin{tabular}{|c|c|c|}
\hline Clonotype (5'-3') & Cancer $(\%)$ & Control $(\%)$ \\
\hline GCCACCAGCAGAACGGCAGGAGAGACCCAGTAC & 0.781 & 0.001 \\
\hline GCCACCAGCAGAGAAGCTAGCGGGATCCAAGAGACCCAGTAC & 0.558 & 0.001 \\
\hline GCCACCAGCAGAGATACTCTAGGCACAGATACGCAGTAT & 0.001 & 2.191 \\
\hline GCCACCAGCAGAGATGGGGGCGGGGTACAAGAGACCCAGTAC & 0.737 & 0.001 \\
\hline GCCACCAGCAGTCCCTCGGGGATCTATGGCTACACC & 1.429 & 0.014 \\
\hline GCCACCAGCGATGATCAGGGGAGTTTTAATGAAAAACTGTTT & 0.748 & 0.001 \\
\hline GCCACCAGTGATATAGCGGGAGAGACCCAGTAC & 1.315 & 0.001 \\
\hline GCCACCAGTGATCGGGACTACTACAATGAGCAGTTC & 1.036 & 0.002 \\
\hline GCCACCAGTGATTCATCGGATTACGAGCAGTAC & 0.501 & 0.033 \\
\hline GCCACCAGTGATTGGGACAGCCCGACCTACGAGCAGTAC & 0.514 & 0.001 \\
\hline GCCACCAGTGATTTGCGGGGACAGGGCCAAGAGACCCAGTAC & 0.896 & 0.001 \\
\hline GCCACCAGTGATTTGGCCAGGCATTATGGCTACACC & 0.650 & 0.001 \\
\hline GCCACCAGTGATTTTAGGGGCCAACCCCAAGAGACCCAGTAC & 1.053 & 0.008 \\
\hline GCCACCAGTGATTTTGATTCGGGACTTTCGGGGACCCAGCAC & 0.656 & 0.001 \\
\hline GCCACCAGTGATTTTGATTCGGGACTTTCGGGGACCCAGTAC & 17.886 & 0.027 \\
\hline GCCACCAGTGATTTTGGAGGGATCCAAGAGACCCAGTAC & 0.595 & 0.001 \\
\hline GCCACCTCAGGGGACGGCTATGGCTACACC & 0.869 & 0.001 \\
\hline GCCAGCACCCTCTGGGGGAACACTGAAGCTTTC & 0.001 & 1.601 \\
\hline GCCAGCAGCTTTGGGAGCGAGCAGTAC & 0.564 & 0.001 \\
\hline GCCAGCAGGGAATTCGGACAGGGGGCAAAGGCTTTC & 1.972 & 0.001 \\
\hline GCCAGCAGTGAAAGCGGGAGTAGTGCAGATACGCAGTAT & 2.047 & 0.001 \\
\hline GCCAGCAGTGAAGGGGGGCAGGGGCGGCAGACCCAGTAC & 0.574 & 0.001 \\
\hline GCCAGCAGTTCCGCAACGTACCGATACACC & 1.303 & 0.009 \\
\hline GCCAGCATATTCGCGGTAGAGACTGAAGCTTTC & 0.001 & 0.533 \\
\hline GCCAGCCAGGGGTTCGGGGAGCTGTTT & 0.811 & 0.001 \\
\hline GCCAGCCTTCCCGAGACTAGCGGGAGCCACGAGCAGTAC & 0.532 & 0.001 \\
\hline GCCAGTACCCATACGGGTCGAGTGGACGGCTACACC & 0.557 & 0.001 \\
\hline GCCAGTAGTATCAATCTTAACGGCGAGCAGTAC & 0.840 & 0.002 \\
\hline GCCAGTAGTATCGGGACAGGGGGCGCCGGGGGCTACACC & 0.839 & 0.001 \\
\hline GCCAGTGGGGAGGGCCGGACCGGGGAGCTGTTT & 2.025 & 0.001 \\
\hline GCCTCGCGGGAGGGCCCCACCGGGGAGCTGTTT & 0.710 & 0.001 \\
\hline GCCTGGAGTATTTGGACTAACACTGAAGCTTTC & 0.001 & 4.212 \\
\hline
\end{tabular}

in the control sample (data not shown). With the exception of TRBJ2-2P (0.01 vs. $0.01 \%)$, the other 13 TRBJ gene segments usage frequencies displayed extreme variation between the cancer sample and control sample. These data indicated that there might be clonal expansion, in particular for the $\mathrm{V}$ and $\mathrm{J}$ gene families, in the cancer sample.

To compare the expression difference of the V-J pairs between the cancer and control samples, seven special V-J pairs were obtained in the cancer sample and 132 special V-J pairs in the control sample; there were 483 shared V-J pairs between the cancer and control samples. However, the seven special V-J pairs in the cancer sample and 132 special V-J pairs in the control sample demonstrated lower usage frequency $(<1 \%)$, whereas for the 483 shared $\mathrm{V}-\mathrm{J}$ pairs, $23 \mathrm{~V}-\mathrm{J}$ pairs were found in the cancer sample and $22 \mathrm{~V}-\mathrm{J}$ pairs in the control sample that exhibited a high usage frequency (>1\%) (Table VIII). Among these, $10 \mathrm{~V}-\mathrm{J}$ pairs were overlapping, and the other V-J pairs revealed extreme variation between the cancer sample and control sample. For example, TRBV24-1-TRBJ2-5 (23.67\%) exhibited the highest expression in the cancer sample, with a low expression in the control sample (0.75\%). Conversely TRBV30-TRBJ1-1 (4.90\%) displayed the highest expression in the control sample, whereas its expression was very low in the cancer sample $(0.22 \%)$. Taken together, these data indicated that specific V-J dominant pairs were dominant in the cancer sample.

\section{Discussion}

Intrinsic and extrinsic factors are important in the development of bladder cancer; however, the exact pathogenesis is still not fully understood. TILs are strictly correlated with patient prognosis and provide the theoretical basis for gene-immunological therapy in bladder cancer (19). Pichler et al revealed that the tumour microenvironment influences the therapeutic response to $\mathrm{BCG}$, which may permit individualized treatment (7). However, most studies on individualized treatment 
Table VII. A summary of the highly expressed shared amino acid sequences $(>0.5 \%)$.

\begin{tabular}{lcc}
\hline Clonotype & Cancer $(\%)$ & Control (\%) \\
\hline ASGEGRTGELF & 2.038 & 0.001 \\
ASIFAVETEAF & 0.001 & 0.538 \\
ASLPETSGSHEQY & 0.535 & 0.001 \\
ASQGFGELF & 0.815 & 0.001 \\
ASREFGQGAKAF & 1.988 & 0.001 \\
ASREGPTGELF & 0.715 & 0.001 \\
ASSEGGQGRQTQY & 0.579 & 0.001 \\
ASSEGRKY & 0.502 & 0.001 \\
ASSESGSSADTQY & 2.063 & 0.001 \\
ASSFGSEQY & 0.565 & 0.001 \\
ASSIGTGGAGGYT & 0.846 & 0.001 \\
ASSINLNGEQY & 0.846 & 0.001 \\
ASSSATYRYT & 1.311 & 0.009 \\
ASTHTGRVDGYT & 0.560 & 0.001 \\
ASTLWGNTEAF & 0.001 & 1.624 \\
ATSDDQGSFNEKLF & 0.755 & 0.001 \\
ATSDFDSGLSGTQH & 0.663 & 0.001 \\
ATSDFDSGLSGTQY & 18.071 & 0.027 \\
ATSDFGGIQETQY & 0.600 & 0.001 \\
ATSDFRGQPQETQY & 1.062 & 0.008 \\
ATSDIAGETQY & 1.329 & 0.001 \\
ATSDLARHYGYT & 0.656 & 0.001 \\
ATSDLRGQGQETQY & 0.903 & 0.001 \\
ATSDRDYYNEQF & 1.042 & 0.001 \\
ATSDSSDYEQY & 0.492 & 0.034 \\
ATSDWDSPTYEQY & 0.517 & 0.001 \\
ATSGDGYGYT & 0.873 & 0.001 \\
ATSRDGGGVQETQY & 0.753 & 0.001 \\
ATSRDTLGTDTQY & 0.001 & 2.241 \\
ATSREASGIQETQY & 0.582 & 0.001 \\
ATSRTAGETQY & 0.789 & 0.001 \\
ATSSPSGIYGYT & 1.437 & 0.014 \\
AWSIWTNTEAF & 0.001 & 4.276 \\
\hline & & \\
& & \\
& &
\end{tabular}

are based on changes and molecular typing of T-cell subsets, and only a few studies focus on the sequence information of TCR binding to tumour antigens. T lymphocytes serve an important role in the tumour immune response; they recognize major histocompatibility complex-bound peptides, which are mediated by TCRs. The CDR3 region of TCRs has a special molecular structure, which represents the different populations of T cells. In the present study, multiplex PCR and HTS were used to investigate the T-cell repertoires in cancerous and paracancerous tissues from patients with bladder cancer. A total of 1,081,491 and 551,870 sequences were obtained from the cancer sample and the control sample, respectively, providing substantial evidence regarding the TCR repertoire in bladder cancer.

HECs are crucial in the immunological repertoire and may be the result of physiological responses to pathogens or antigens.

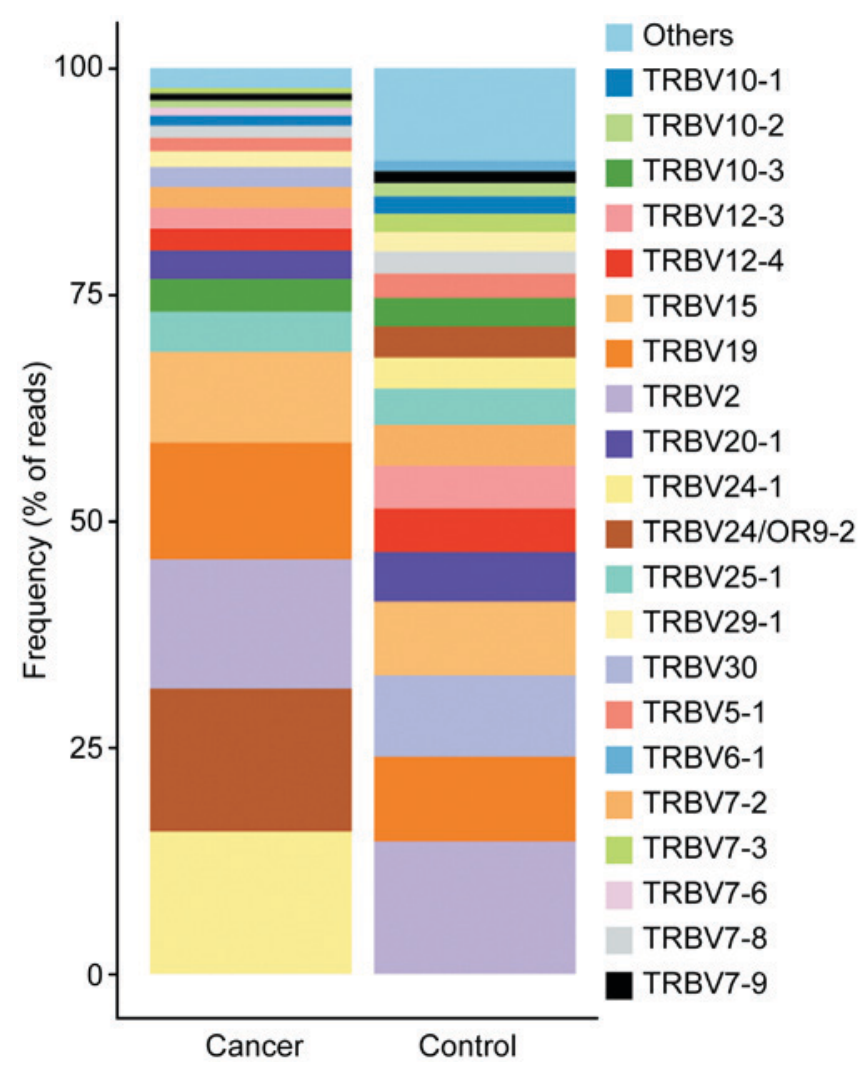

Figure 3. Stacked bar chart for the usage frequencies of the top $20 \mathrm{TRBV}$ gene family segments in the cancer sample and control sample. The $\mathrm{x}$-axis depicts the name of the sample, and the y-axis depicts the frequencies of the TRBV gene subtype, which accounts for the percentage of all the TRBV gene subtypes in the sample. TRBV, T-cell receptor $\beta$ variable.

In the present study, the number of HECs and the contribution of these HECs to the total TCR repertoire were analysed. The TCR repertoire in the cancer sample had a greater HEC $(>0.5 \%$ total TCR analysed) number, HEC ratio, and a higher degree clone percentage $(>0.1 \%)$ than in the control sample. These results suggested that the degree of amplification in cancer tissue was significantly higher than in paracancerous tissue. Furthermore, to better understand the TCR diversity of the cancer and control samples, the Shannon entropy index (20) was used. The results suggested that the diversity of cancer tissues was lower than that of paracancerous tissues. Nakanishi et al similarly demonstrated that the proportion of CDR3 aa sequence accounting for $>0.01 \%$ of the total molecular population in the TILs is significantly higher than in peripheral blood lymphocytes in colorectal cancer (15). In addition, Han et al revealed that the HEC ratio of the TCR $\beta$ chain CDR3 sequence in carcinoma tissues is significantly higher than in the adjacent tissues from patients with hepatitis B virus-associated hepatocellular carcinoma (21). Velotti et al, via high-resolution PCR, determined the CDR3 size lengths of TCR $\beta$ transcripts from patients with bladder cancer, and demonstrated that the number of expanded clones in the cancer tissues is larger than that in peripheral blood (22). This difference may be explained by the prolonged exposure of $\mathrm{T}$ lymphocytes to tumour antigens, leading to a targeted rearrangement of the TCR gene, eventually resulting in significant enrichment of the corresponding clones in the cancer tissues. The identification of bladder cancer-specific HECs may therefore accelerate the screening process of potential novel tumour 
Table VIII. V-J pair usage percentage $(>1 \%)$ in the cancer and control samples.

\begin{tabular}{lc}
\hline A, Cancer & \\
\hline V-J pairs & Usage $(\%)$ \\
\hline TRBV24-1, TRBJ2-5 & 23.67 \\
TRBV15, TRBJ2-5 & 3.79 \\
TRBV19, TRBJ2-2 & 3.78 \\
TRBV19, TRBJ1-2 & 3.27 \\
TRBV2, TRBJ2-3 & 2.65 \\
TRBV2, TRBJ2-7 & 2.29 \\
TRBV24-1, TRBJ2-7 & 2.23 \\
TRBV2, TRBJ1-1 & 2.18 \\
TRBV12-3, TRBJ1-1 & 2.05 \\
TRBV24-1, TRBJ2-1 & 1.89 \\
TRBV2, TRBJ2-5 & 1.82 \\
TRBV2, TRBJ1-5 & 1.79 \\
TRBV24-1, TRBJ1-2 & 1.72 \\
TRBV15, TRBJ1-2 & 1.48 \\
TRBV12-3, TRBJ1-2 & 1.47 \\
TRBV19, TRBJ2-7 & 1.41 \\
TRBV10-3, TRBJ2-7 & 1.38 \\
TRBV15, TRBJ2-7 & 1.37 \\
TRBV19, TRBJ2-5 & 1.32 \\
TRBV2, TRBJ1-2 & 1.26 \\
TRBV15, TRBJ1-4 & 1.24 \\
TRBV25-1, TRBJ1-2 & 1.21 \\
TRBV25-1, TRBJ2-7 & 1.11 \\
\hline & \\
\hline
\end{tabular}

B, Control

\begin{tabular}{lc}
\hline V-J pairs & Usage $(\%)$ \\
\hline TRBV25-1, TRBJ1-4 & 1.57 \\
TRBV15, TRBJ2-7 & 2.14 \\
TRBV19, TRBJ2-5 & 2.13 \\
TRBV19, TRBJ1-2 & 1.43 \\
TRBV20-1, TRBJ2-5 & 1.10 \\
TRBV24-1, TRBJ1-5 & 1.76 \\
TRBV30, TRBJ1-1 & 4.90 \\
TRBV2, TRBJ2-1 & 1.06 \\
TRBV12-3, TRBJ1-2 & 1.00 \\
TRBV25-1, TRBJ1-1 & 1.15 \\
TRBV20-1, TRBJ2-7 & 1.20 \\
TRBV2, TRBJ2-7 & 3.21 \\
TRBV24-1, TRBJ2-7 & 1.07 \\
TRBV12-3, TRBJ2-7 & 1.18 \\
TRBV12-3, TRBJ2-2 & 1.94 \\
TRBV2, TRBJ1-2 & 2.09 \\
TRBV12-3, TRBJ1-1 & 1.12 \\
TRBV19, TRBJ1-1 & 1.89 \\
TRBV2, TRBJ1-1 & 2.85 \\
TRBV2, TRBJ2-5 & 1.63 \\
TRBV15, TRBJ2-3 & 2.69 \\
TRBV7-2, TRBJ2-7 & 1.38 \\
\hline
\end{tabular}

Bold indicates shared V-J pairs. TRBV, T-cell receptor $\beta$ variable. antigens. This screening is critical to better understand the TCR repertoire, and may represent a novel diagnostic tool for patients with bladder cancer.

In the present study, analysis of the expression levels of TRBV, TRBD and TRBJ repertoires was systematically performed and revealed significant differences in the cancer tissues compared with the paracancerous tissues. In addition, 13 identified V-J pairs were highly expressed $(>1 \%)$ in the cancerous tissue, whereas their expression was very low in the paracancerous tissue. The high expression of one or more TCR BV subfamilies in the cancer tissues from patients with bladder cancer may be associated with the immune pathogenesis of the disease. Therefore, the present study provided a theoretical foundation for further investigations on the immune pathogenesis and gene-immunological therapy of bladder cancer. In the absence of disease, TCR BV is rearranged randomly, and T cells exhibit a positive polyclonal state. However, in the case of disease, prolonged stimulation by specific antigens gives rise to a selective rearrangement and excessive abnormal cloning of one or more TCR BV subfamilies; excessive cloning of the cloned $\mathrm{T}$ cell may thus inhibit cloning of other T cells $(23,24)$. Wang et al reported a preferential use of TRBV and TRBJ genes in cancerous tissues compared with adjacent tissues in breast cancer (25). A recent study identified specific V-J pairs that may distinguish the TCR repertoires of patients with liver cancer from healthy subjects. These specific V-J pairs may therefore be used as novel biomarkers in liver cancer (21).

In conclusion, the present study demonstrated that deep analysis of the TCR families from tissue samples may be performed using a HTS platform. This may be a powerful novel approach to evaluate the complexity and diversity of the T-cell immune repertoire in bladder cancer. However, the sample size of this study was small, which did not allow a complete description of the diversity of the TCR $\beta$ CDR3 repertoire in various types of bladder cancer. A better understanding of the functional basis of the TCR $\beta$ CDR3 clonalities is crucial, and further studies based on larger sample sizes, using tools such as NetMHC and SYFPEITHI, are required to identify certain particular peptides including signal transducer and activator of transcription 3 and glutathione S-transferase alpha 1. This may represent a novel biomarker for the detection, prognosis and development of gene-immunological therapy of bladder cancer.

\section{Acknowledgements \\ Not applicable. \\ Funding}

This study was supported by grants from The Scientific and Technology Project of Guangdong Province (grant no. 2016A050503009) and the Science and Technology Plan of Shenzhen (grant no. JCY20160422164313440).

\section{Availability of data and material}

The datasets used and/or analysed during the current study are available from the corresponding author on reasonable request. 


\section{Authors' contributions}

JM, YD and WS conceived and designed the experiments. JM, GS, PZ and SL performed the experiments. JM, SL, MO and ZC analysed the data. GS, CZ and FLC provided reagents, materials and analysis tools, and acquired the data. JM and WS wrote the paper.

\section{Ethics approval and consent to participate}

This study passed the discussion and approval of the Ethics Committee of Guilin No. 181 Hospital and Shenzhen People's Hospital of Guangdong. All patients consented to participate in the study and for their tissues to be used, as specified in the Declaration of Helsinki.

\section{Patient consent for publication}

Not applicable.

\section{Competing interests}

The authors declare that they have no competing interests.

\section{References}

1. de la Cruz-Merino L, Grande-Pulido E, Albero-Tamarit A and Codes-Manuel de Villena ME: Cancer and immune response: Old and new evidence for future challenges. Oncologis 13 : 1246-1254, 2008.

2. Gooden MJ, de Bock GH, Leffers N, Daemen T and Nijman HW: The prognostic influence of tumour-infiltrating lymphocytes in cancer: A ystematic review with meta-analysis. Br J Cancer 105: 93-103, 2011.

3. Delyon J, Mateus C, Lefeuvre D, Lanoy E, Zitvogel L, Chaput N, Roy S, Eggermont AM, Routier E and Robert C: Experience in daily practice with ipilimumab for the treatment of patients with metastaticmelanoma: An early increase in lymphocyte and eosinophil counts is associated with improved survival. Ann Oncol 24: 1697-1703, 2013.

4. Lee AH, Gillett CE, Ryder K, Fentiman IS, Miles DW and Millis RR: Different patterns of inflammation and prognosis in invasive carcinoma of the breast. Histopathology 48: 692-701, 2006.

5. Lipson EJ, Sharfman WH, Drake CG, Wollner I, Taube JM, Anders RA, Xu H, Yao S, Pons A, Chen L, et al: Durable cancer regression off-treatment and effective reinduction therapy with an anti-PD-1 antibody. Clin Cancer Res 19: 462-468, 2013.

6. Brahmer JR, Tykodi SS, Chow LQ, Hwu WJ, Topalian SL, Hwu P, Drake CG, Camacho LH, Kauh J, Odunsi K, et al: Safety and activity of anti-PD-L1 antibody in patients with advanced cancer. N Engl J Med 366: 2455-2465, 2012.

7. Pichler R, Fritz J, Zavadil C, Schäfer G, Culig Z and Brunner A: Tumor-infiltrating immune cell subpopulations influence the oncologic outcome after intravesical Bacillus Calmette-Guérin therapy in bladder cancer. Oncotarget 7: 39916-39930, 2016.

8. Davis MM and Bjorkman PJ: T cell antigen receptor genes and T cell recognition. Nature 334: 395-402, 1988.

9. Rubtsova K, Scott-Browne JP, Crawford F, Dai S, Marrack P and Kappler JW: Many different Vbeta CDR3s can reveal the inherent MHC reactivity of germline-encoded TCR V regions. Proc Natl Acad Sci USA 106: 7951-7956, 2009.
10. Arstila TP, Casrouge A, Baron V, Even J, Kanellopoulos J and Kourilsky P: A direct estimate of the human alphabeta $\mathrm{T}$ cell receptor diversity. Science 286: 958-961, 1999.

11. Serratì S, De Summa S, Pilato B, Petriella D, Lacalamita R, Tommasi S and Pinto R. Next-generation sequencing: Advances and applications in cancer diagnosis. Onco Targets Ther 9: 7355-7365, 2016

12. Calis JJ and Rosenberg BR: Characterizing immune repertoires by high throughput sequencing: Strategies and applications. Trends Immunol 35: 581-590, 2014.

13. Gerlinger M, Quezada SA, Peggs KS, Furness AJ, Fisher R, Marafioti T, Shende VH, McGranahan N, Rowan AJ, Hazell S, et al: Ultra-deep T cell receptor sequencing reveals the complexity and intratumour heterogeneity of T cell clones in renal cell carcinomas. J Pathol 231: 424-432, 2013.

14. Weng WK, Armstrong R, Arai S, Desmarais C, Hoppe R and Kim YH: Minimal residual disease monitoring with high-throughput sequencing of $\mathrm{T}$ cell receptors in cutaneous $\mathrm{T}$ cell lymphoma. Sci Transl Med 5: 214ra171, 2013.

15. Nakanishi K, Kukita Y, Segawa H, Inoue N, Ohue M and Kato K: Characterization of the $\mathrm{T}$ cell receptor beta chain repertoire in tumor-infiltrating lymphocytes. Cancer Med 5: 2513-2521, 2016.

16. Lefranc MP: Immunoglobulin and $\mathrm{T}$ cell receptor genes: IMGT(®) and the birth and rise of immunoinformatics. Front Immunol 5: 22, 2014.

17. Hou X, Lu C, Chen S, Xie Q, Cui G, Chen J, Chen Z, Wu Z, Ding Y, Ye P, et al: High throughput sequencing of $\mathrm{T}$ cell antigen receptors reveals a conserved TCR repertoire. Medicine (Baltimore) 95: e2839, 2016.

18. Sui W, Hou X, Zou G, Che W, Yang M, Zheng C, Liu F, Chen P, Wei X, Lai L and Dai Y: Composition and variation analysis of the TCR $\beta$-chain CDR3 repertoire in systemic lupus erythematosus using high-throughput sequencing. Mol Immunol 67: 455-464, 2015.

19. Lipponen PK, Eskelinen MJ, Jauhiainen K, Harju E and Terho R: Tumour infiltrating lymphocytes as an independent prognostic factor in transitional cell bladder cancer. Eur J Cancer 29A: 69-75, 1992.

20. Magurran AE: Measuring biological diversity. John Wiley \& Sons, 2013

21. Han Y, Liu X, Wang Y, Wu X, Guan Y, Li H, Chen X, Zhou B, Yuan Q, Ou Y, et al: Identification of characteristic TRB V usage in HBV-associated HCC by using differential expression profiling analysis. OncoImmunology 4: e1021537, 2015.

22. Velotti F, Chopin D, Gil-Diez S, Maille P, Abbou CC, Kourilsky P and Even J: Clonality of tumor-infiltrating lymphocytes in human urinary bladder carcinoma. J Immunother 20: 470-478, 1997.

23. Long SA, Khalili J, Ashe J, Berenson R, Ferrand C and Bonyhadi M: Standardized analysis for the quantification of Vbeta CDR3 T-cell receptor diversity. J Immunol Methods 317: 100-113, 2006

24. Miqueu P, Guillet M, Degauque N, Doré JC, Soulillou JP and Brouard S: Statistical analysis of CDR3 length distributions for the assessment of T and B cell repertoire biases. Mol Immunol 44: 1057-1064, 2007

25. Wang T, Wang C, Wu J, He C, Zhang W, Liu J, Zhang R, Lv Y, Li Y, Zeng X, et al: The different $T$ cell receptor repertoires in breast cancer tumors, draining lymph nodes, and adjacent tissues. Cancer Immunol Res 5: 148-156, 2017.

This work is licensed under a Creative Commons Attribution-NonCommercial-NoDerivatives 4.0 International (CC BY-NC-ND 4.0) License. 